\title{
Investigation into the factors that influence inverse bulging effect during sheet hydro-mechanical deep drawing
}

\author{
Lihui Lang ${ }^{1}$, Yao Wang ${ }^{1}$, Kui $\mathrm{Li}^{1}$, Shiyue $\mathrm{Li}^{1}$, Jianmin Zhang ${ }^{2}$, and Quanda Zhang ${ }^{1}$ \\ ${ }^{1}$ School of Mechanical Engineer and Automation, Beihang University, Beijing 100191, China \\ ${ }^{2}$ Tianjin Tianduan Press Co., LTD, Tianjin 301700, China
}

\begin{abstract}
The factors that influence inverse bulging effect during sheet hydro-mechanical deep drawing are especially researched in this paper. According to the different inverse bulging process, two modes can be singled: the initial inverse bulging (IIB) and the local inverse bulging (LIB). IIB includes two parameters: inverse bulging height ratio $\left(H_{I b} / t\right)$ and inverse bulging pressure ratio $\left(P_{I b} / t\right)$. LIB is influenced by IIB and has a direct relationship with liquid chamber pressure in the forming process. The optimal inverse bulging parameters of hemispherical bottom cylindrical part and flat bottom cylindrical part are obtained by numerical simulation. Process parameters including the clearance between the punch and the blank holder and the blank holder entrance radius that have a large influence on inverse bulging effect are optimized, so as to make inverse bulging effect behave better in hydroforming process. Finally, the accuracy of the numerical simulation results was verified by experiments.
\end{abstract}

\section{Introduction}

As one of the important processing technology in modern industry, sheet hydroforming has gained increasing interest in the aerospace and automotive manufacturing. In sheet hydroforming, many parameters influence the process, such as the blank holding gap, the blank holding force, the die entrance radius, liquid chamber pressure and the inverse bulging effect [1-5]. In these parameters, the inverse bulging effect plays an important and decisive role in the sheet hydroforming. Compared with previous publications, this paper focuses on the factors that influence inverse bulging effect during sheet hydromechanical deep drawing especially, such as the clearance between the punch and the blank holder and the blank holder entrance radius, and tries to find the appropriate process parameters to make the inverse bulging effect play a greater role in sheet hydroforming. In this paper, the inverse bulging is divided into two types: initial inverse bulging (IIB) and local inverse bulging (LIB) (as shown in Fig. 1). The initial inverse bulging (IIB) has two functions: the first is to build the liquid chamber pressure at the beginning stage of hydroforming, and the second is to change the stress state of sheet around the punch nose to avoid the defect of fracture at the beginning of forming. The local inverse bulging (LIB) can be seen as a soft drawbead which can decrease the circumferential compressive stress at die entrance region and produce biaxial tensile stress to avoid the defect of wrinkling. Meanwhile, the larger LIB can reduce the returning its previous state phenomenon of the sheet at punch nose and improves forming

This is an Open Access article distributed under the terms of the Creative Commons Attribution License 4.0, which permits unrestricted use, distribution, and reproduction in any medium, provided the original work is properly cited. 


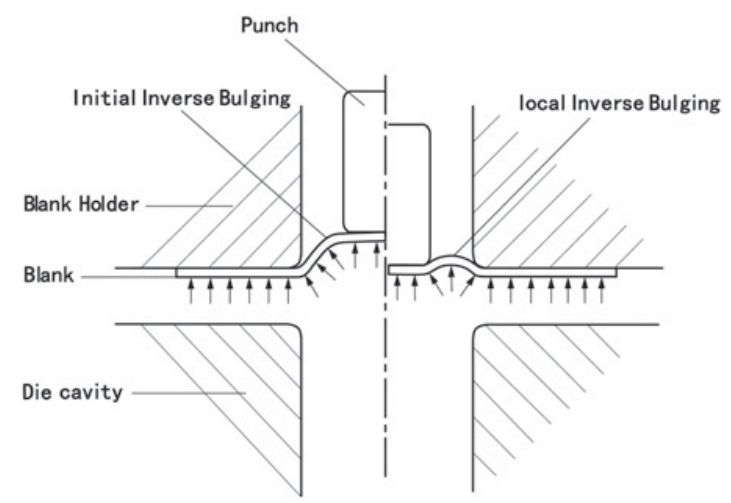

Figure 1. The inverse bulging hydro-mechanical deep drawing.

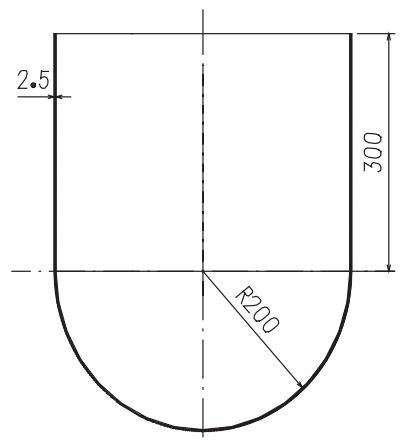

a) Hemispherical bottom cylindrical part

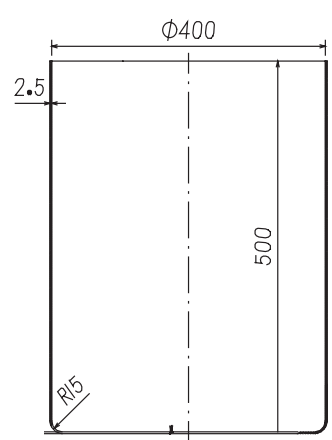

b) Flat bottom cylindrical part

Figure 2. The geometric shape and size of specimens.

accuracy at wall and bottom of parts. The main parameters of IIB are inverse bulging height and inverse bulging pressure. LIB is influenced by IIB and has a direct relationship with liquid chamber pressure in the forming process. In order to avoid the influence of sheet thickness on inverse bulging effect and be consistent with other parameters research method, the inverse bulging height ratio $H_{I b} / t$ and the inverse bulging pressure ratio $P_{I b} / t$ are adopted to research inverse bulging effect in this paper [6].

\section{Basic parameters}

The material used was the $2.5 \mathrm{~mm}$ thick stainless steel SUS304. Its property is shown in Table 1 . The inverse bulging effect and its effect factors of hemispherical bottom cylindrical part and flat bottom cylindrical part are researched respectively. The geometric shape and size of specimens are shown in Fig. 2. The hemispherical diameter is $\Phi 400 \mathrm{~mm}$ with height is $500 \mathrm{~mm}$. The diameter of flat bottom cylindrical part is also $\$ 400 \mathrm{~mm}$ with height is $500 \mathrm{~mm}$ and the bottom corner radius is $15 \mathrm{~mm}$. Considering the deformation feature, trimming and process allowance, the circular plate whose diameter is $900 \mathrm{~mm}$ was chosen as the blank. The die inside diameter is $406.4 \mathrm{~mm}$ and the die entrance radius is $25 \mathrm{~mm}$. The gap between the die and the blank holder is fixed in the experiment and the value is $2.75 \mathrm{~mm}$. All the experimental work was carried out on the 4500 ton sheet hydroforming equipment. The liquid pressure in the die cavity is controlled by a proportional pressure relief valve whose maximum pressure can reach $100 \mathrm{MPa}$. 
Table 1. Mechanical property for stainless steel SUS304.

\begin{tabular}{|c|c|c|c|c|c|}
\hline $\begin{array}{c}\text { Yield } \\
\text { strength } \\
\boldsymbol{\sigma}_{s}(\mathbf{M p a})\end{array}$ & $\begin{array}{c}\text { Tensile } \\
\text { strength } \\
\boldsymbol{\sigma}_{b}(\mathrm{MPa})\end{array}$ & $\begin{array}{c}\text { Strength } \\
\text { factor } \\
\boldsymbol{K}(\mathbf{M P a})\end{array}$ & $\begin{array}{c}\text { Strain } \\
\text { hardening } \\
\text { factor } \boldsymbol{n}\end{array}$ & $\begin{array}{c}\text { Elongation } \\
\boldsymbol{\delta}(\%)\end{array}$ & $\begin{array}{c}\text { Elasticity } \\
\text { modulus } \\
\boldsymbol{E}(\mathbf{G P a})\end{array}$ \\
\hline 205 & 529 & 980 & 0.502 & 40 & 207 \\
\hline
\end{tabular}

Table 2. Numerical simulation scheme ( $\mathrm{t}$ is the sheet thickness).

\begin{tabular}{|l|l|}
\hline \multirow{2}{*}{ Blank holder entrance radius $(\mathbf{m m}): 20(8 \mathrm{t})$} & $\begin{array}{l}\text { Clearance between the punch and the blank } \\
\text { holder }(\mathbf{m m})\end{array}$ \\
\cline { 2 - 2 } & $1.25(0.5 \mathrm{t}) ; 2.5(1 \mathrm{t}) ; 3.75(1.5 \mathrm{t}) ; 5(2 \mathrm{t}) ; 6.25(2.5 \mathrm{t})$ \\
\hline $\begin{array}{l}\text { Clearance between the punch and the blank } \\
\text { holder }(\mathbf{m m}): 3.75(1.5 \mathrm{t})\end{array}$ & Blank holder entrance radius $(\mathbf{m m})$ \\
\cline { 2 - 2 } & $5(2 \mathrm{t}) ; 15(6 \mathrm{t}) ; 20(8 \mathrm{t}) ; 25(10 \mathrm{t}) ; 32.5(13 \mathrm{t})$ \\
\hline
\end{tabular}

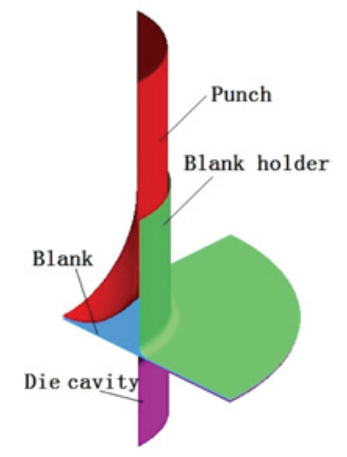

a) Hemispherical bottom cylindrical part

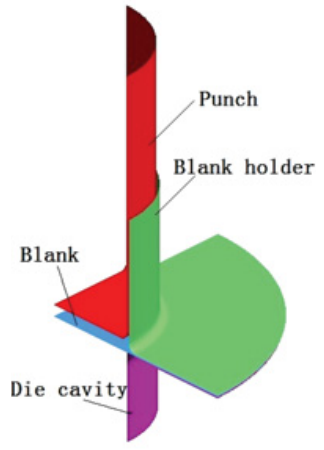

b) Flat bottom cylindrical part

Figure 3. The FEM models.

\section{Simulation and analysis}

\subsection{FEM model and scheme}

Finite element analysis software Dynaform5.8.1/LS-DYNA3D was used in the numerical simulation. Because of the axi-symmetric property of parts, only quarter of the physical model was used and finite element models were shown in Fig. 3. All tools were modeled using a rigid shell element. The blank was modeled using the B-T shell element. A friction coefficient of 0.12 was used for the interfaces between the punch and the blank, and 0.05 for that between the blank and the blank holder and between the blank and the die, respectively. The gap between the die and the blank holder was fixed and the value was $2.75 \mathrm{~mm}$. The numerical simulation scheme was shown in Table 2.

\subsection{Influence of inverse bulging effect on forming process}

The optimal inverse bulging parameters of hemispherical bottom cylindrical part and flat bottom cylindrical part were obtained through repeated numerical simulations. For the hemispherical bottom cylindrical part, when the inverse bulging height ratio was 2 , the inverse bulging pressure ratio was 0.8 and the liquid chamber pressure was $80 \mathrm{MPa}$, its formability was best. The specimens thinning ratio distributions with and without inverse bulging were contrasted as shown in Fig. 4. As can be seen, the part can be once-formed, and the main thinning all occurs at the initial stage of forming. Then, the decreasing rate of thickness slows down along with the hydro-mechanical deep drawing, and the part thickness hardly changes after the intermediate stage of forming. Compared to no-initial inverse 


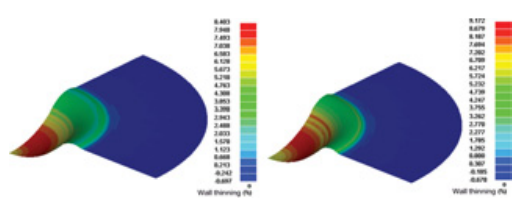

a) The initial stage

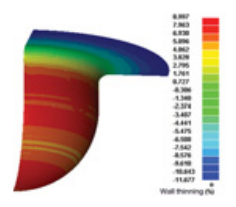

b) The intermediate stage
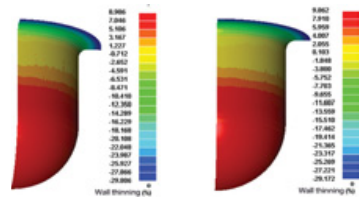

c) The later stage

Figure 4. The thinning ratio distributions of hemispherical bottom cylindrical part (inverse bulging/no-inverse bulging).

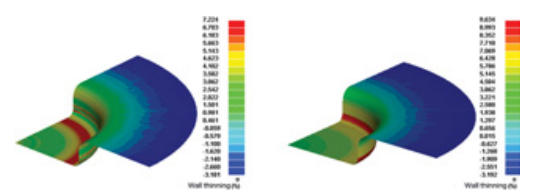

a) The initial stage

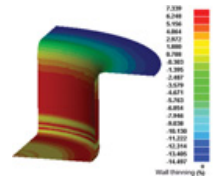

b) The intermediate stage

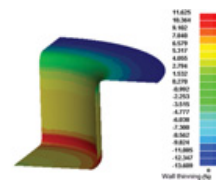

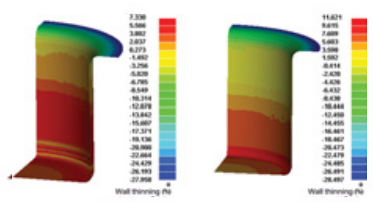

c) The later stage

Figure 5. The thinning ratio distributions of flat bottom cylindrical part (inverse bulging/no-inverse bulging).

bulging, the wall thinning ratio of the part is decreased by $8.9 \%$ with initial inverse bulging. For the flat bottom cylindrical part, when the inverse bulging height ratio was 10 , the inverse bulging pressure ratio was 2 and the liquid chamber pressure was $90 \mathrm{MPa}$, its formability was best. The specimens thinning ratio distributions with and without inverse bulging were contrasted as shown in Fig. 5. Similar to the hemispherical bottom cylindrical part, the specimen thickness thins largely at the initial stage of forming, and the wall thinning ratio is controlled effectively by the inverse bulging effect at the initial stage. As the numerical simulations of these two parts shown, the inverse bulging effect improves the formability of the parts mainly by improving the formability at the initial stage of forming.

\subsection{Influence of clearance between the punch and the blank holder on inverse bulging}

The $1.25 \mathrm{~mm}(0.5 \mathrm{t}), 2.5 \mathrm{~mm}(1 \mathrm{t}), 3.75 \mathrm{~mm}(1.5 \mathrm{t}), 5 \mathrm{~mm}(2 \mathrm{t})$ and $6.25 \mathrm{~mm}(2.5 \mathrm{t})$ were chosen respectively for the clearance between the punch and the blank holder. Wall thickness distributions of the two specimens obtained by numerical simulation were shown in Fig. 6 and Fig. 7 respectively. The contrast of maximum wall thinning ratio of the two specimens was shown in Fig. 8. For the hemispherical bottom cylindrical part, with increasing of the clearance between the punch and the blank holder, the wall thickness of the specimen and its distribution uniformity also increase correspondingly with improvement in formability. According to the characteristic of the specimen material flow in forming process, the smaller clearance is, the lower thickness reduction is at the initial stage of inverse bulging. However, with the hydro-mechanical deep drawing, the smaller clearance leads to the material flow shortage for the central region of hemisphere bottom and the increasing seriousness in thickness reduction. For the flat bottom cylindrical part, the maximum wall thinning ratio of the specimen with different clearance between the punch and the blank holder is $7.67 \%, 7.36 \%, 7.33 \%, 7.66 \%$ and $8.69 \%$ respectively. When the clearance is $2.5 \mathrm{~mm}$ (1t) or $3.75 \mathrm{~mm}(1.5 \mathrm{t})$, the wall thinning ratio of the specimen is lower with better in formability. According to the characteristic of the specimen material flow in forming process, during the initial inverse bulging, the smaller the clearance is, the more difficult the flow up of the material would be and the more severe the thickness reduction at the bottom of the flat bottom cylindrical part would be. When the punch went down, the larger clearance couldn't make the blank form the reverse bending material storage area (as shown in Fig. 9), and the hardenability degree of the blank in this area is lower. Finally, the blank in this area would flow and become the thinnest 


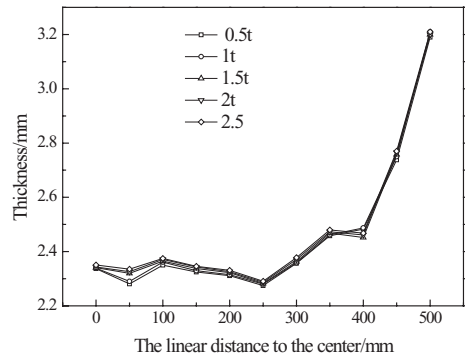

Figure 6. Thickness distributions of hemispherical bottom cylindrical part.

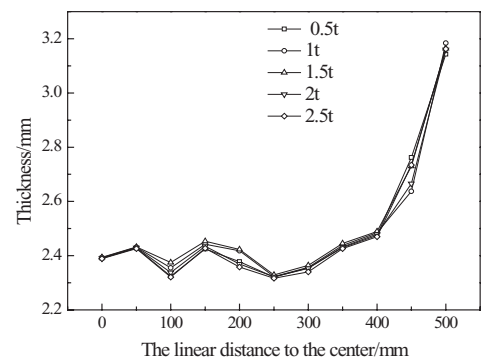

Figure 7. Thickness distributions of flat bottom cylindrical part.

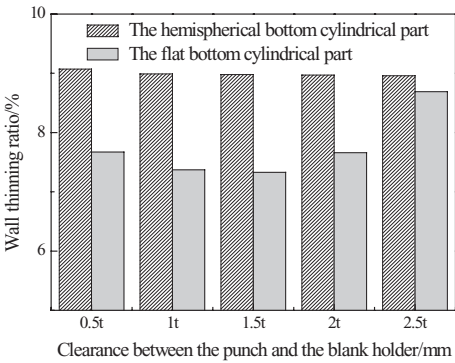

Figure 8. Contrast of maximum thinning ratio of the two specimens.

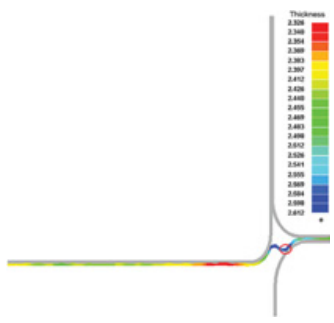

a) $1.25 \mathrm{~mm}(0.5 \mathrm{t})$

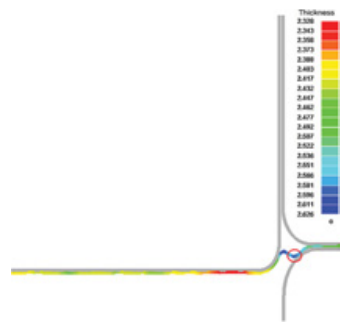

b) $2.5 \mathrm{~mm}(1 \mathrm{t})$

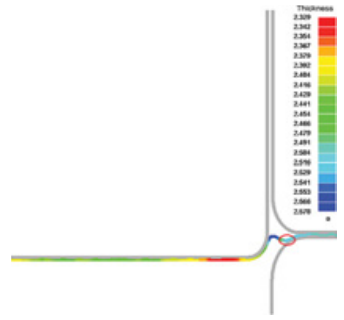

c) $3.75 \mathrm{~mm}(1.5 \mathrm{t})$

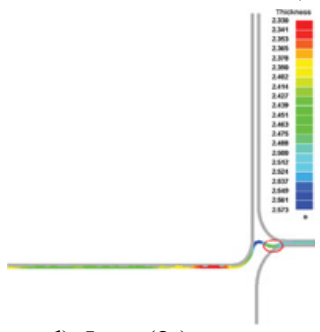

d) $5 \mathrm{~mm}(2 \mathrm{t})$

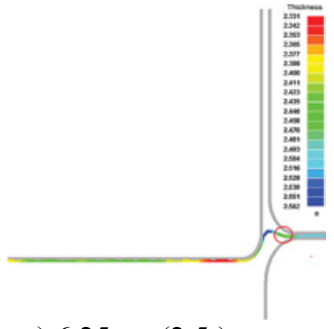

e) $6.25 \mathrm{~mm}(2.5 \mathrm{t})$

Figure 9. Reverse bending material storage area with different clearance between the punch and the blank holder.

portion of wall thickness in the subsequent deep drawing process. Therefore, a reasonable clearance between the punch and the blank holder can improve the formability of sheet.

\subsection{Influence of the blank holder entrance radius on inverse bulging}

The $5 \mathrm{~mm}(2 \mathrm{t}), 15 \mathrm{~mm}(6 \mathrm{t}), 20 \mathrm{~mm}(8 \mathrm{t}), 25 \mathrm{~mm}(10 \mathrm{t})$ and $32.5 \mathrm{~mm}(13 \mathrm{t})$ were chosen respectively for the blank holder entrance radius. Wall thickness distributions of the two specimens were shown in Fig. 10 and Fig. 11 respectively. The contrast of maximum wall thinning ratio was shown in Fig. 12. For the hemispherical bottom cylindrical part, the maximum wall thinning ratio is $8.73 \%, 8.93 \%, 8.98 \%, 9.01 \%$ and $9.23 \%$ respectively. As can be seen, the larger blank holder entrance radius is not beneficial for the formability attributes of the sheet. According to the characteristic of the specimen material flow in forming process, the center of hemisphere bottom was the most severe thinning position at the initial stage of forming. The smaller blank holder entrance radius is, the more material storage around the rim of hemisphere bottom center would be, the more sufficient material flow from the rim of bottom to the center of bottom is along with the punch went down, and the more obvious the effect that controlling 


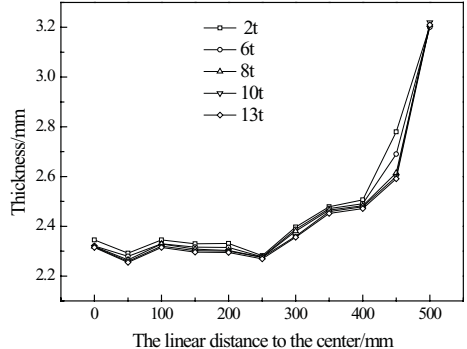

Figure 10. Thickness distributions of hemispherical bottom cylindrical part.

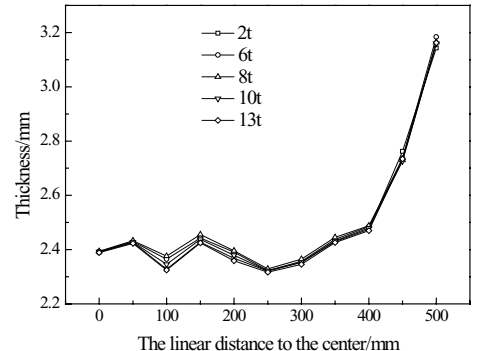

Figure 11. Thickness distributions of flat bottom cylindrical part.

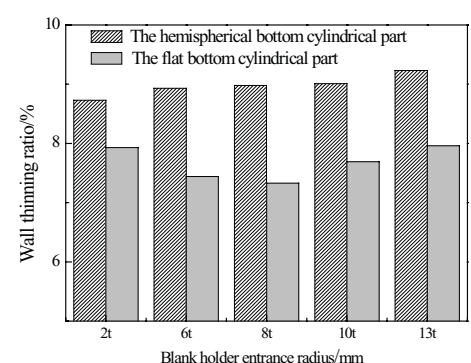

Figure 12. Contrast of maximum thinning ratio of the two specimens.

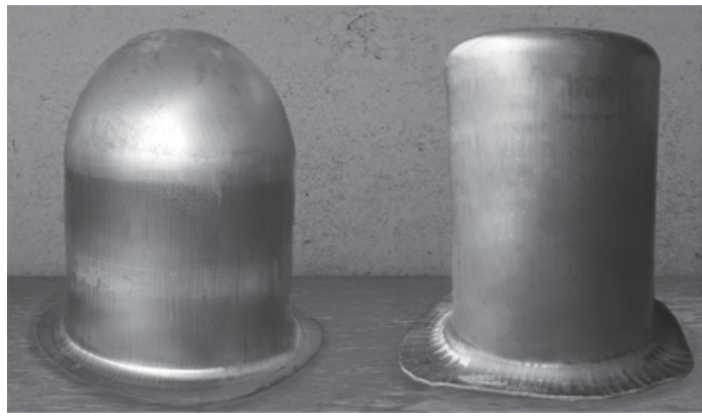

Figure 13. The experiment specimens.

the wall thickness thinning is at the center of bottom. For the flat bottom cylindrical part, the maximum wall thinning ratio is $7.93 \%, 7.44 \%, 7.33 \%, 7.69 \%$ and $7.96 \%$ respectively. When the blank holder entrance radius is $20 \mathrm{~mm}(8 \mathrm{t})$, the wall thinning ratio of the specimen is lowest with best in formability. In forming process, the flow up of the material is restricted by the too small blank holder entrance radius during the initial inverse bulging, which leads to the severe thickness reduction at the bottom of the specimen. When the punch went down, the larger blank holder entrance radius couldn't make the blank form the reverse bending material storage area, and the hardenability degree of the blank in this area is lower. Finally, the blank in this area would flow and become the thinnest portion of wall thickness in the subsequent deep drawing process. In conclusion, too small or too large blank holder entrance radius both is not good for the part forming.

\section{Experiment result and analysis}

In order to verify the accuracy of the numerical simulation, inverse bulging hydro-mechanical deep drawing experiments of stainless steel hemisphere bottom cylinder part and flat bottom cylindrical part were carried out on the $4500 \mathrm{~T}$ sheet hydroforming equipment. The specimens were obtained by applying the optimal inverse bulging parameters, the reasonable clearance between the punch and the binder and the suitable binder entrance radius, as shown in Fig. 13. The specimens thickness distributions measured by the ultrasonic thickness measuring unit were compared with the result of the numerical simulation, as shown in Fig. 14. It can be seen that the experiments results are basically coincide with that of simulation. 


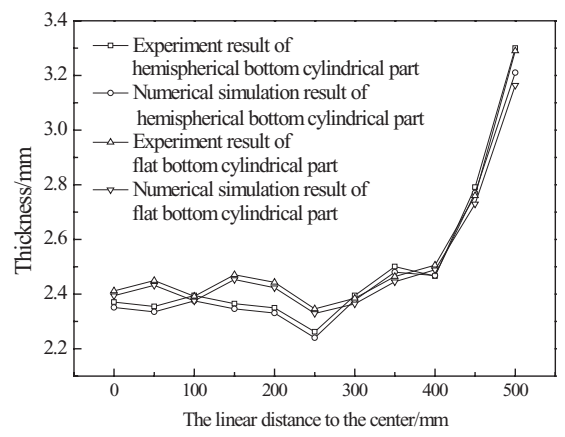

Figure 14. Comparison of experiment results and numerical simulation results.

\section{Conclusions}

1. The inverse bulging effect improves the formability of the parts mainly by improving the formability at the initial stage of forming.

2. For the hemispherical bottom cylindrical part, the larger clearance between the punch and the blank holder is beneficial for the formability attributes of the sheet. For the flat bottom cylindrical part, too small or too large the clearance both is not good for the part forming.

3. For the hemispherical bottom cylindrical part, the smaller blank holder entrance radius is beneficial for the formability attributes of the sheet. For the flat bottom cylindrical part, too small or too large blank holder entrance radius both is not good for the part forming.

\section{References}

[1] S.K. Singh, D.R. Kumar, J. Mater. Process. Tech 204 (2008) 169-178

[2] H.S. Halkaci, M. Turkoz, M. Dilmec, J. Mater. Process. Tech 214 (2014) 1638-1646

[3] L.H. Lang, Z.R. Wang et al, J. Mater. Process. Tech 151 (2004) 165-177

[4] B. Meng, M. Wan et al., Int. J. Mech. Sci 77 (2013) 217-226

[5] T. Hama, K. Matsushima et al., ISIJ International 49 (2009) 1736-1743

[6] L.H. Lang, J. Danckert, K.B. Nielsen, Int. J. Mach. Tool. Manu 44 (2004) 649-657 